\title{
Cryptanalysis and Improvement of RFID Ownership Transfer Protocol
}

\author{
Xiuqing Chen ${ }^{1}$, Qiang Zhao ${ }^{1}$, Tianjie $\mathrm{Cao}^{2}$ and Jingxuan Zhai ${ }^{2}$ \\ ${ }^{1}$ School of Medicine Information, Xuzhou Medical College, Xuzhou, Jiangsu \\ 221000, P.R. China \\ ${ }^{2}$ School of Computer, China University of Mining and Technology, Xuzhou, China \\ xiuqingchen@126.com
}

\begin{abstract}
The widespread use of Radio Frequency Identification (RFID) technologies help to trace a large number of commodity and share the tag information in the supply chain system. However, many ownership transfer protocols are subject to various attacks. We analyze the security of two protocols. Even if the designers claim that their protocols are security, we find that their schemes suffer from forward traceability attacks and tracing attacks. In addition, we show that a weak attacker can retrieve the secrets of the tag with a probability 1 in Kardaş et al.'s protocol. To resist against these attacks, we present an improved scheme based on Kardaş et al.'s protocol by adopting the new key-update mechanism. In the end, we show the enhanced versions provides the forward and backward untraceable security properties.
\end{abstract}

Keywords: RFID; ownership transfer protocol; key disclosure attack; forward untraceable; backward untraceable

\section{Introduction}

Radio Frequency Identification (RFID) technologies have been extensively deployed in the ubiquitous domain, such as luggage box tracing, supply chain system and health care medical system [1-3]. Low-cost RFID tags in supply chain system will be better choices for the shopkeepers and manufacturers that currently pay close attention to increase the efficiency of the identification compared with barcode technology. RFID tag technology can apply to different kinds of products that desire researchers to design how to implement the real-world functions. Moreover, this efficient technology can guarantee that the tag identificates automatically the legitimate product.

Despite those advantages, the security and the privacy of the tag owner are the main concern in the rapid and widespread application of this distinguished technology. The existing ownership transfer protocols for supply chain system have respective advantages. Among them, the security and the privacy of the entities (tag and owner) have played the important role. However, the later security analysis carried out on many schemes [4-6] have showed obvious weaknesses. This has motivated researchers improve the vulnerable schemes in order to protect against various attacks. For example, the key disclosure attack in protocol is an attack that the current messages of the tag and monitored and the current and next run's keys should be revealed by an attacker. Besides, forward and backward untraceable is introduced in [7], where the attacker run the current protocol and obtain the future and the past secret keys of the target tag.

*Corresponding author 
When an attacker knows the related keys of the target tag, s/he is able to trace its location. It is necessary for researcher to present RFID ownership transfer protocol, to amend the weaknesses and enhance its security level, analyze the security of the improved scheme in security model.

The main goal of this paper is to point out the weaknesses of two protocols [8, 14] which are liable to tracing attack and forward untraceable attack. Then, we modify one protocol and achieve secure ownership transfer in the further. We demonstrate that the modified version meets backward untraceability against the new owner and forward untraceability against the old owner. Moreover, we also show that our protocol provides forward and backward untraceability using the formal model.

The outline of the paper is as follows. In Section 2, the related work and adversary model are introduced, respectively. Section 3 and 4 analyses the weaknesses in Doss et al.'s scheme [14] and Karda's et al.'s protocol, respectively. In Section 5, our enhanced protocol based on Karda's et al.'s protocol and safety analysis of the scheme is showed in detail. In Section 6, we propose the conclusion.

\section{Preliminaries}

For simplicity, the notations used through the paper are defined in Table 1.

Table 1. Notations and Descriptions

\begin{tabular}{ll}
\hline Notations & Descriptions \\
\hline$R, T, \mathrm{DB}, A d v^{+}$ & The reader,the tag, server database, the strong adversary. \\
$\oplus, \|$ & XOR and concatenation operation. \\
$h, H$ & Hash functions. \\
$v a l_{1}, v a l_{2}$ & Validation messages from $R$ and $T$. \\
$N_{a}, N_{b}, \gamma$ & Random numbers. \\
$r_{1}, r_{2}, v_{i}$ & Random nonces created by $h\left(N_{a}\right), h\left(N_{b}, K\right), h\left(K, r_{1}, r_{2}\right)$. \\
$K, K^{\text {old }}, K^{\text {old } 2}$ & Shared secrets between $T$ and $R$. \\
$r, n$ & The keys in the tag. \\
$T I D ; h(T I D)$ & The identifier of tag; The hash value of identifier. \\
\hline
\end{tabular}

In this paper, we adopt the oracles in the Vaudenay model [9]. The following definitions and oracles are used to analyze security property of the RFID protocol.

- CreateTag $(I D)$ creates the free tag with $I D$ using $\operatorname{SetupTag}\left(I D_{i}\right)$.

- DrawTag() stochastically selects a tag from the free tags to the drawn tags as vtag.

- Free(vtag) gets back the tag vtag to the set of free tags.

- Launch ()$\rightarrow \pi$ activates a new protocol $\pi$.

- SendReader-Tag $\left(\boldsymbol{m}_{\boldsymbol{R}}, \boldsymbol{\pi}\right) \rightarrow \boldsymbol{m}_{\boldsymbol{R}}$. The $R^{i}$ sends $\boldsymbol{m}_{\boldsymbol{R}}$ from $R^{i}$ to tag and obtains the response $\boldsymbol{m}{ }^{\prime}{ }_{\boldsymbol{R}}$.

- SendTag ( $\boldsymbol{m}, \boldsymbol{v t a g}) \rightarrow \boldsymbol{m}$ '. The tag $\boldsymbol{v t a g}$ transmits the message $\boldsymbol{m}$ and responds with $\boldsymbol{m}$ '.

- $\operatorname{Result}(\pi)$ returns 1 when protocol instance $\pi$ is successfully executed or 0 otherwise.

- Corrupt(vtag) $\rightarrow$ keys. The adversary knows the secrets of the tag vtag.

This paper mainly introduces two types of attacker ability: narrow-strong attacker and weak attacker. To be special, the former is in the forward and backward untraceable analysis, and the latter is in tracing attack analysis.

Definition 1 (Forward untraceable). It is impossible for narrow-strong $A d v^{+}$to trace the tag at the $i^{\text {th }}\left(i^{\prime} \geqslant i+2\right)$ run, when $A d v^{+}$knows the $i^{\text {th }}$ secrets of the tag and misses the $(i+1)^{\text {th }}$ run in the protocol.

Definition 2 (Backward untraceable). When narrow-strong $A d v^{+}$corrupts the $i^{\text {th }}$ keys of the tag, s/he can not speculate the $(i-1)^{\text {th }}$ secrets of the tag. 
Definition 3 (Weak indistinguishability). When the weak attacker chooses the target tag $T^{*}$ to test, s/he obtains the target outputs. If the attacker tests with diverse tags and monitors the different outputs, s/he can differentiate at least one tag is not the target tag.

\section{Cryptanalysis of Doss et al.'s Protocol}

We introduce Doss et al.'s protocol in Figure. 1. The collaborative authentication phase is as follows:

Step 1. Reader to Tag

The reader sends the messages ( hello, $s$ ) to the tag.

Step 2. Tag to Reader

The tag calculates $x=h(T I D) \oplus r \oplus s \oplus t$. The tag also computes $x^{\prime}=x^{2} \bmod n ; x^{\prime \prime}=\left(x^{2}\right)^{2}$ $\bmod n$ and $t^{\prime \prime}=\left(t^{2}\right)^{2} \bmod n$. The tag forwards $\left(x ', t t^{\prime \prime}\right)$ to the reader.

In order to simplify the analysis steps, the steps 3-6 in Figure 1 are omitted here. The tracing attack in Doss et al.'s protocol has three phases.

1) The testing phase:

The attacker chooses the target tag $T^{*}$, and sets $s=0$. Then, s/he sends ( hello, 0 ) to $T^{*}$, and monitors the outputs $(* x ", * t)$. We can obtain the following equation $(* x " \oplus$ $* t ')=(h(T I D) \oplus r)^{4} \bmod n$. Therefore, the computed value $(* x, " \oplus * t ')$ should be fixed owing to the fixed keys $h(T I D), r$ and $n$ for $T^{*}$.

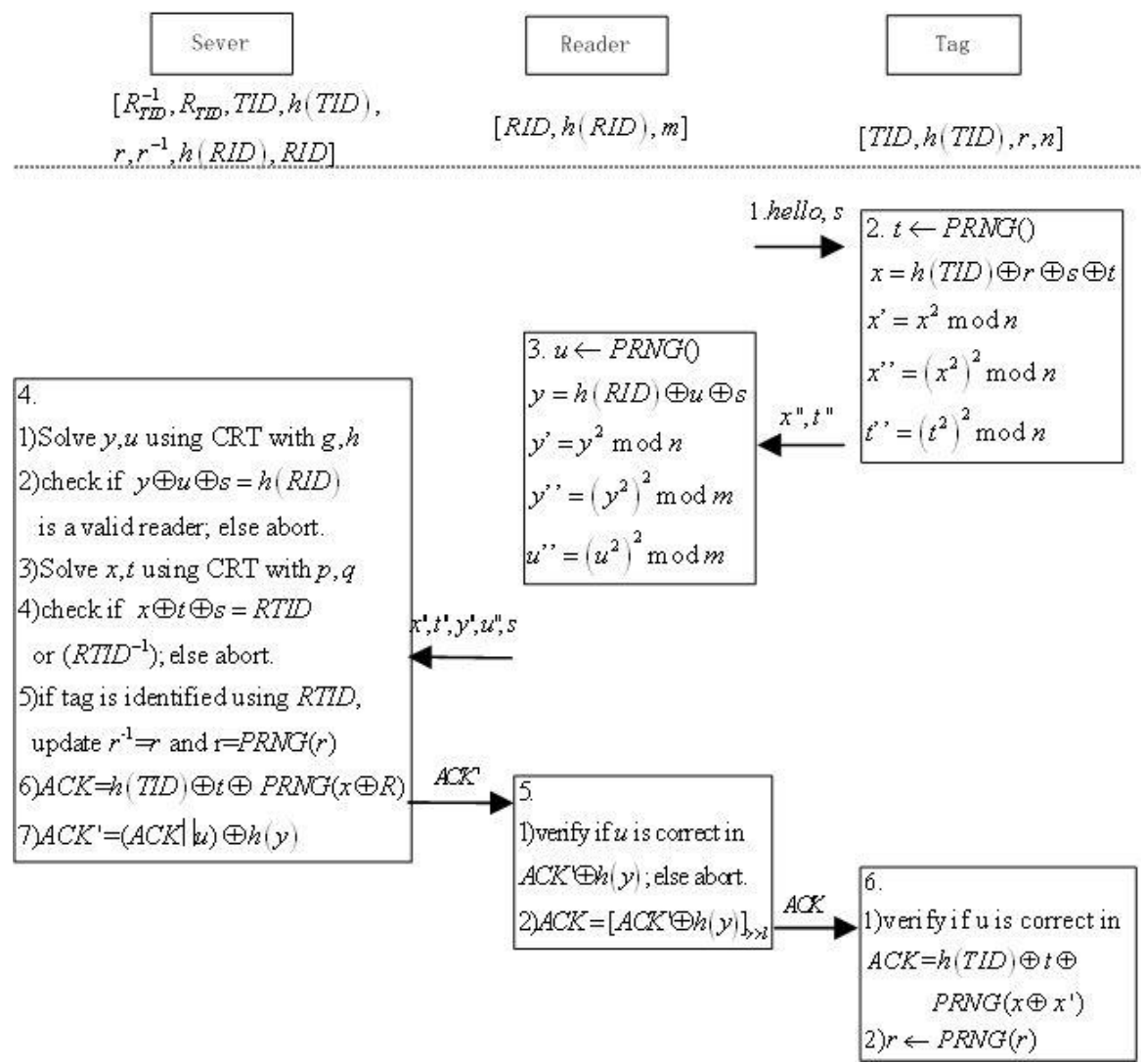

Figure 1. The Collaborative Authentication Phase in Doss et al.'s Protocol

2) The tracing attack phase:

We assume that the tag set $\left(T^{0}, T^{1}, \ldots, T^{i}\right)$ includes $T^{*}$ and the counterfeit tag $T$ with its keys $\left(h\left(T I D^{\prime}\right), r^{\prime}\right)$, where $h\left(T I D^{\prime}\right) \oplus r^{\prime}=h(T I D) \oplus r$. The attacker sends (hello, $\left.s=0\right)$ to each tag $T^{i}$ and obtains the output $\left({ }^{i}{ }^{\prime},{ }^{\prime},{ }^{i} t^{\prime}\right)$. 
3) The decision phase:

The attacker computes the XORed value $\left({ }^{i} x " \oplus^{i} t\right.$ '") compared with the value $(* x, \oplus$

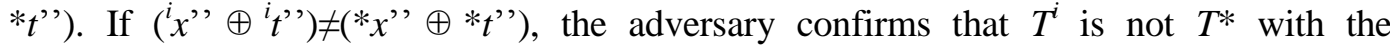
probability 1; If $\left({ }^{i} x " \oplus^{i} t^{\prime}{ }^{\prime}\right)=\left(* x ' \oplus * t^{\prime} '\right)$, the adversary is not able to make sure that $T^{i}$ is $T^{*}$ or the counterfeit tag $T$, since $\left({ }^{i} x^{\prime \prime} \oplus^{i} t^{\prime} '\right)=h\left(T I D D^{\prime}\right) \oplus r^{\prime}=h(T I D) \oplus r$. Therefore, Doss et al.'s protocol does not meet the property of weak indistinguishability and suffers from the tracing attack.

\section{Cryptanalysis of Kardaş et al.'s Protocol}

We give a detail process of Kardaş et al.'s protocol as follows:

1) The initialization phase:

The registrar stores three keys $(K, S, L)$. It also computes $v^{2} l_{1}=h(L, K), v a l_{2}=h(S)$. The registrar creates $\left(K, S, v a l_{1}, K^{\text {old } 1}, K^{\text {old } 2}, S^{\text {old }}, v^{\text {old }} l^{\text {ol } 1}, h(K)\right)$ in the DB and arranges $(K, L$, $v a l_{2}$, state $)$ to the tag and $\left(K, S, v a l_{1}\right)$ to the reader, respectively.

2) The authentication phase:

The steps are described below in Figure2.

Step 1. $R_{i}$ cteates a random number $N_{a}$ and calculates $r_{1}=h\left(N_{a}\right)$. Then it sends $r_{1}$ to the $T_{i}$.

Step 2. $T_{i}$ cteates a random number $N_{b}$ and calculates $r_{2}=h\left(N_{b}, K\right)$. If the value state is equal to 0 , it calculates $\gamma=h(K)$. If not, $T_{i}$ generates a random number $\gamma$. Later, $T_{i}$ calculates $v_{1} \| v_{2}=H\left(K, r_{1}, r_{2}\right)$. After that, the $T_{i}$ computes message $M_{1}=v_{1} \oplus L$. Finally, $T_{i}$ transmits $r_{2}$, $M_{1}$ and $\gamma$ to the $R_{i}$.

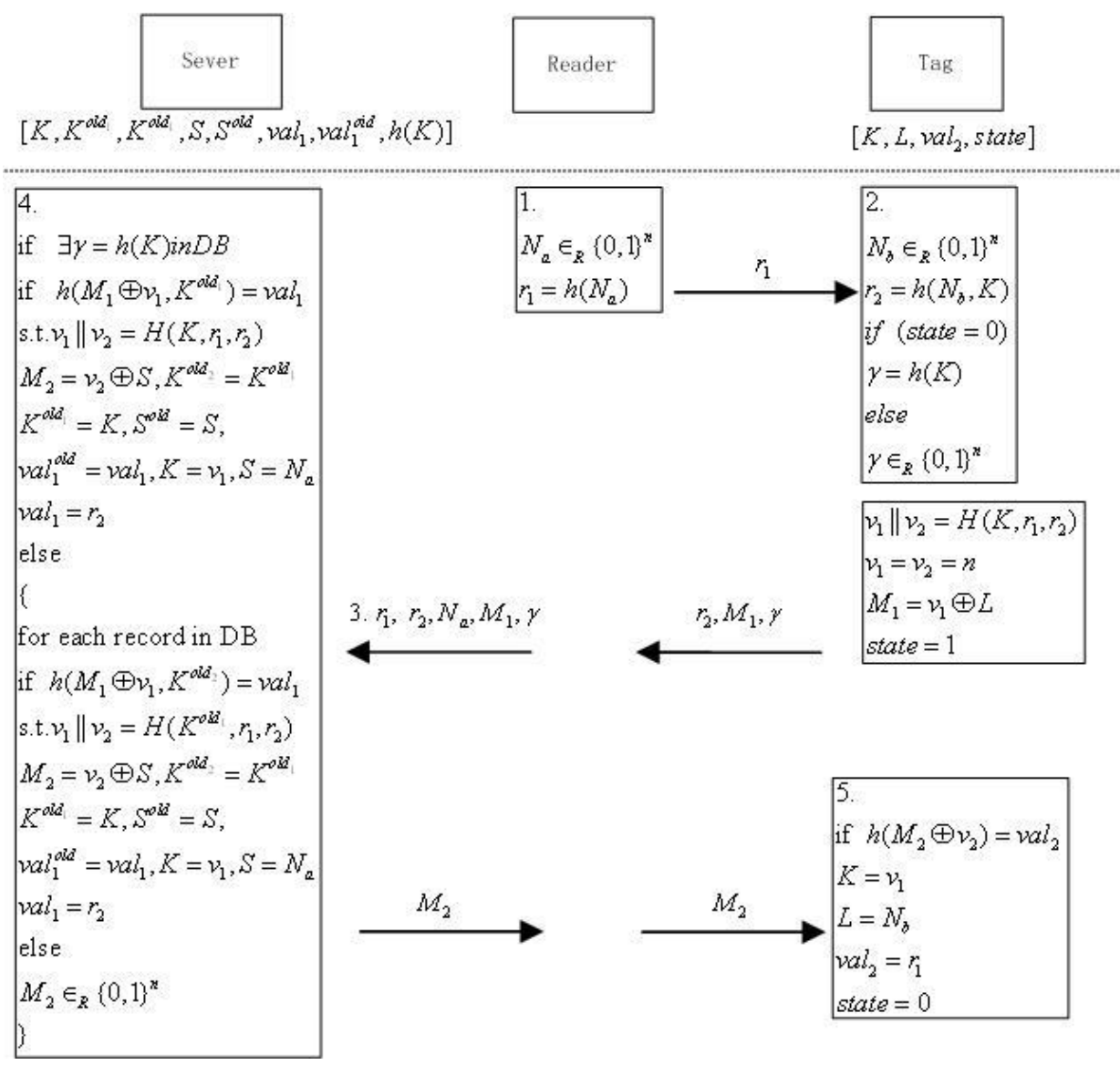

Figure 2. Kardas s et al.'s Protocol 
Step 3. The $R_{i}$ transmits $N_{a}, r_{1}, r_{2}, M_{1}$, and $\gamma$ to DB.

Step 4 . The DB firstly verifies that $h(K) ?=\gamma$. The DB performs an exhaustive search among all tags.

It computes $v_{1} \| v_{2}=H\left(K, r_{1}, r_{2}\right)$ and $h\left(M_{1} \oplus v_{1}, K\right)$. DB checks $h\left(M_{1} \oplus v_{1}, K^{o l d 1}\right) ?=v a l_{1}$. When the equation holds, DB calculates $M_{2}=v_{2} \oplus S$ and then sends $M_{2}$ to the $R_{i}$. After that, it updates $K^{\text {old } 2}=K^{\text {old } 1}, K^{\text {old } 1}=\mathrm{K}, S^{\text {old }}=S, \mathrm{val}^{\text {old } 1}=v a l_{1}, K=v_{2}, S=N_{a}$, and $v a l_{1}=r_{2}$. If no match is found, then the DB performs another an exhaustive search among all tags in the database. In this time, it computes $v_{1} \| v_{2}=\mathrm{H}\left(K^{o l d 1}, r_{1}, r_{2}\right)$ and it checks $h\left(M_{1} \oplus v_{1}, K^{o l d 2}\right)$ ?= val $l^{o l d}$. If one match is found, DB computes $M_{2}=v_{2} \oplus S$ and sends $M_{2}$ to the $T_{i}$. It updates $K$ $=v_{2}, S=N_{a}$, and $v a l_{1}=r_{1}$.

However, when there is no match, in order to prevent the adversary to verify $M_{1}$ for $r_{1}$ and $r_{2}$, the DB generates the nonce $M_{2}$ and sends it to the $R_{i}$.

Step 5. $R_{i}$ forwards $M_{2}$ to the $T_{i}$. Upon receiving $M_{2}, T_{i}$ computes $\mathrm{h}\left(M_{2} \oplus v_{2}\right)$ and checks whether it is equal to $v a l_{2}$. If equal, then it updates $K=v_{2}, L=N_{b}$, and $v a l_{2}=r_{1}$.

$\mathrm{Karda}_{s}$ set al.'s proposed an efficient and private RFID authentication protocol supporting ownership transfer (see Figure.2). They claimed that their protocol have achieved forward and backward untraceable security. However, we point out their scheme suffers from forward untraceable attack and key exposure attack. Then, we show the attacks in detail.

The leakage of the tag's keys totally threatens the RFID system. In particular, the adversary can monitor, store and modify the transmitted messages. Alternatively, the adversary can counterfeit the messages of the tag. We introduce two attacks on the protocol as follows:

\subsection{Tracing Attack on Karda s set al.'s Protocol Under the Weak Attacker}

Since $r_{1}, r_{2}$ and $N_{a}$ are sent in the open, an adversary (the malicious old owner) can trace the future states of $T_{i}$ that tag's key $v a l_{2}=r_{1}$ which is owned by the new owner. Meanwhile, the $(i+1)^{\text {th }}$ keys $\left\{v a l_{1}, S\right\}$ stored in DB are revealed by reason that the keys $\left\{v a l_{1}, S\right\}$ depended on $\left\{r_{2}, N_{a}\right\}$ respectively in the key-update mechanism.

\subsection{Forward Traceability Attack on Protocol under the Strong Attacker}

The protocol runs the $i^{\text {th }}$ session between $T^{i}$ and $R^{i}$, the attacker monitors the $i^{\text {th }}$ messages $\left\{{ }^{i} r_{1},{ }^{i} r_{2},{ }^{i} M_{1},{ }^{i} M_{2}\right\}$. After the successfully run, the updated keys of $T$ is ${ }^{i+1} K$, ${ }^{i+1} L,{ }^{i+1} \mathrm{val}_{2},{ }^{i+1}$ state $\}$. An adversary (the old owner) knows the old key ${ }^{0} K$ and monitors two successive run of the protocol and obtains all exchanged messages $\left\{{ }^{0} r_{1},{ }^{0} r_{2},{ }^{0} M_{1},{ }^{0} M_{2}\right\}$ and $\left\{{ }^{1} r_{1},{ }^{1} r_{2},{ }^{1} M_{1},{ }^{1} M_{2}\right\}$ between tag and reader. Since ${ }^{0} r_{1}$ and ${ }^{0} r_{2}$ are sent in the open, knowing the construction of $H$, an adversary (the old owner) can obtain the tag's key ${ }^{1} v a l_{2}={ }^{0} r_{1}$ and compute ${ }^{0} v_{1} \|{ }^{0} v_{2}=H\left({ }^{0} K,{ }^{0} r_{1},{ }^{0} r_{2}\right)$, then ${ }^{1} K={ }^{0} v_{1}$. The computational process of key ${ }^{1} K$ as well as ${ }^{2} K$ is above process. Since the key ${ }^{2} K={ }^{1} v_{1}$ can be computed from ${ }^{1} v_{1} \|{ }^{1} v_{2}=H\left({ }^{1} K,{ }^{1} r_{1},{ }^{1} r_{2}\right)$ using ${ }^{0} v_{1}$ in the second run, the adversary can compute ${ }^{1} L={ }^{1} v_{1} \oplus$ ${ }^{1} M_{1}$. Next, the key ${ }^{1}$ state can be speculation with a probability $1 / 2$. Therefore, the old owner can obtain all keys of $\operatorname{tag}^{1}\left\{{ }^{1} K,{ }^{1} L,{ }^{1} v a l_{2},{ }^{1}\right.$ state $\}$ and trace the second run messages of tag.

\section{The Improvement of Kapoor et al.'s Protocol}

In this section, we present a authentication scheme which is the improved version of the protocol proposed in [8]. In our protocol, we achieve the forward untraceable and backward untraceable property whereas computational expense and Communication cost is low in the system. Then, the initialization and the authentication phases are same as the original protocol. The enhanced protocol is summarized in Figure 3. 
Soon afterwards, we modify Karda s et al.'s protocol to make the enhanced protocol against the attacks described in the previous section. To improve the security of the protocol, we assume that all tags and DB are equipped with new update mechanism. The DB updates the keys $K=v_{1} \oplus r_{2}, S=N_{a} \oplus v_{2}$, and $v a l_{1}=r_{2} \oplus v_{2}$, while the tag renews the secrets $K=v_{1} \oplus r_{2}, L=N_{b} \oplus v_{2}$, and $v a l_{2}=r_{1} \oplus v_{2}$.

\subsection{The Formal Privacy Proof}

In the paper, we provide untraceable security analysis in Vaudenay's model with a strong attacker.

Theorem 1 The improved protocol is forward untraceable.

Moreover, in order to meet forward untraceable, the encrypted value $v_{2}$ should be encrypted in the key-update mechanism. After a scheme successfully runs $i^{\text {th }}$ round, the updated secrets of $T_{i}$ as follows.

${ }^{i+1} K^{x}={ }^{i} v_{1}^{x} \oplus r_{2},{ }^{i+1} L^{x}={ }^{i} v_{2}^{x} \oplus N_{b}^{x},{ }^{i+1}{ }{ }^{2} l_{2}^{x}={ }^{i} v_{2}^{x} \oplus r_{1}$.

Even if $A d v^{+}$corrupts the $\left\{{ }^{i} K,{ }^{i} L,{ }^{i} v a l_{2},{ }^{i}\right.$ state $\}$ and monitors the messages $\left\{{ }^{i} r_{1},{ }^{i} r_{2},{ }^{i} M_{1}\right.$, $\left.{ }^{i} M_{2}\right\}$ in the $i^{\text {th }}$ run, the computation of ${ }^{i+1} K^{x}={ }^{i+2} v_{1}^{x} \oplus r_{2}$ is impossible. The attacker has no access to the random numbers $\left\{{ }^{i+2} r_{1}^{x},{ }^{i+2} r_{2}^{x}\right\}$.

Proof

CreateTag $\left(I D_{0}\right)$, CreateTag $\left(I D_{1}\right)$

vtag $\leftarrow$ DrawTag $\left(I D_{c}\right)$, where $c \epsilon\{0,1\}$

${ }^{i} K,{ }^{i} L,{ }^{i}$ val $_{2},{ }^{i}$ state $\leftarrow$ corrupt() at time interval $[i-1, i+1]$

${ }^{i} r_{1} \leftarrow$ SendReader-tag $(\pi$, Init , ID)

${ }^{i} r_{2},{ }^{i} M_{1},{ }^{i} \gamma \leftarrow$ SendTag -Reader $\left(\right.$ vtag,$\left.{ }^{i} r_{1}\right)$

${ }^{i} r_{1},{ }^{i} r_{2},{ }^{i} N_{a},{ }^{i} M_{1},{ }^{i} \gamma \leftarrow$ SendReader-DB $\left(\pi,{ }^{i} r_{2},{ }^{i} M_{1},{ }^{i} \gamma\right)$

Free(vtag)

$\operatorname{Vtag}^{x} \leftarrow$ DrawTag $\left(I D_{x}\right)$ between 2 tags

$A d v^{+}$chooses another time interval $i=[i+2]$

${ }^{i+1} K^{x}={ }^{i} v_{1}^{x} \oplus r_{2},{ }^{i+1} L^{x}={ }^{i} v_{2}^{x} \oplus N_{b}^{x},{ }^{i+1}{ }^{i} a l_{2}^{x}={ }^{i} v_{2}^{x} \oplus r_{1}$. $\pi^{i+2} \leftarrow$ Launch

${ }^{i+2} r_{1} \leftarrow$ SendReader-tag $\left(\pi^{i+2}\right.$, Init $)$

${ }^{i+2} r_{2},{ }^{i+2} M_{1},{ }^{i+2} \gamma \leftarrow$ SendTag-Reader $\left(v \operatorname{tag}^{x},{ }^{i+2} r_{2}\right)$

${ }^{i} r_{1},{ }^{i} r_{2},{ }^{i} N_{a},{ }^{i} M_{1},{ }^{i} \gamma \leftarrow$ SendReader-DB $\left(\pi,{ }^{i} r_{2},{ }^{i} M_{1},{ }^{i} \gamma\right)$

The queries is finished, acknowledges receipt of $\tau\left(\right.$ vtag $\left.^{\prime}\right)=I D_{x}$.

Even if the attacker knows the $i^{\text {th }}$ keys and messages, s/he cannot calculate $(i+1)^{\text {th }}$ keys $K$ without the random number $r_{1}$ and $r_{2}$. The reason is that computation of ${ }^{i+1} K={ }^{i+1}\left[v_{1} \oplus r_{2}\right]$ depends on the unknown value $r_{2}$ and calculated value ${ }^{i+1} v_{1}$.

$A d v^{+}$cannot distinguish between $v$ tag and $v t a g^{x}$ without the $(i+2)^{\text {th }}$ keys $\left\{K^{x}, L^{x}, v a l_{2}^{x}\right\}$. Since $A d v^{+}$cannot compute $\left\{{ }^{i+2} r_{2},{ }^{i+2} M_{1},{ }^{i+2} \gamma\right\}$ without $(i+2)^{\text {th }}$ keys, $A d v^{+}$is not trivial and $\left\{A d v_{A}^{\text {Upriv }}(k), A d v_{A}^{\text {Fupriv }}(k)\right\}=0<<\varepsilon$.

Since $A d v^{+}$is allowed to initiate protocol and monitor the information between $T$ and $R$, s/he cannot trace the $(i+2)^{\text {th }}$ messages. Therefore, the improved protocol is forward untraceable. 

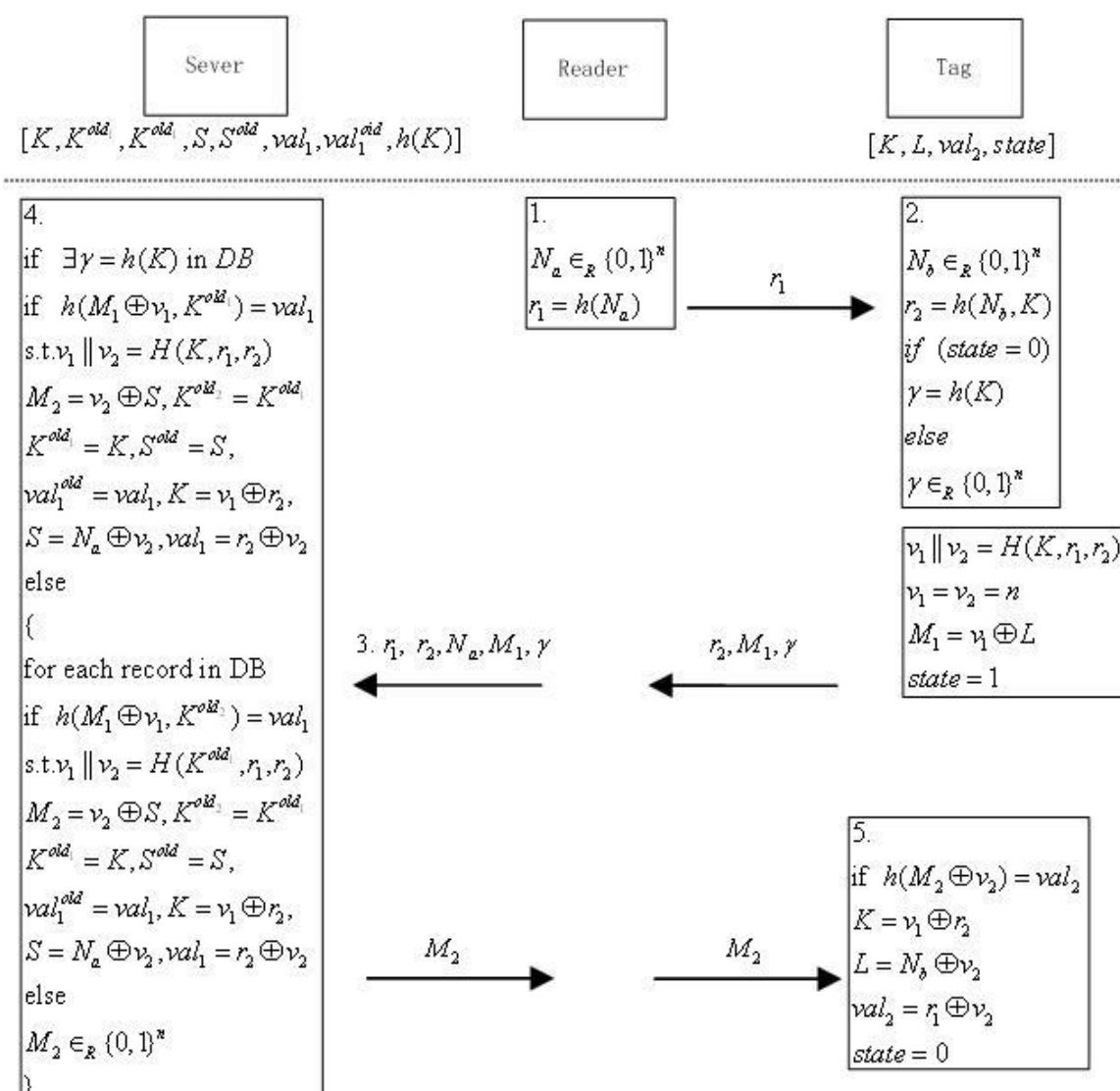

Figure 3. The Improved Protocol

Theorem 2 The improved protocol is backward untraceable.

Proof

$A d v^{+}$is not able to differentiate between vtag and $v \operatorname{tag}^{x}$ without the $(i-1)^{\text {th }}$ secrets $\left\{K^{x}\right.$, $\left.L^{x}, v a l_{2}^{x}\right\}$. Due to the irreversibility of hash function, $A d v^{+}$cannot compute $\left\{K_{i-1}^{x}, P_{i-1}^{x}, C_{i-1}^{x}\right\}$ with $\left\{K_{i}^{x}, P_{i}^{x}, C_{i}^{x}\right\}, A d v^{+}$is trivial and $\left\{A d v_{A}^{\text {BUpriv }}(k)\right\}=0<<\varepsilon$.

We demonstrate that our protocol meets forward and backward untraceable in the Vaudenay's model. Next, we show that our scheme provides security properties.

\subsection{Security Analysis}

1) Resistance against secret parameters disclosure and replay attack

Since the random number $r_{1}$ are sent in plain text, the attacker is not able to reveal the current key of the tag and can calculate the updated key using the public information and the known functional structure of the tag. Nevertheless, the revised protocol resolves the questions by enhancing the key-update mechanism. Even if the attacker monitors the $i^{\text {th }}$ messages, s/he cannot calculate the keys of the target tag.

2) Resistance against tag impersonation attack

In order to impersonate the legitimate tag, the attacker should create a valid $M_{1}$ in our protocol. For the case where state $=0$, the attacker cannot produce a valid $v_{1}$ because the nonce $r_{2}$ and $\gamma=h(K)$ are generated by the tag and the attacker can guess it with a negligible probability. For the case where state $=1$, the random numbers $r_{2}$ and $r \neq h(K)$ are suggested by the tag and the attacker is able to calculate $M_{1}$ and $\gamma$ with a negligible probability.

Nevertheless, the attacker can intercept the data from the reader to the DB (the tag) and relay them later when the tag keys always remain old, tag impersonate attack fails.

3) Resistance against DoS attack 
Since the DB preserves the old and new keys, does not de-synchronize the tag and the reader in the next run. Hence, to de-synchronize a specific tag, the adversary can modify the interactive messages between the reader and the tag such that the tag successfully authenticates the reader with a negligible probability.

4) Resistance against traceability attack

If the attacker replays $r_{1}$, obtains the different messages $r_{2}, M_{1}$ and $\gamma$ from the target tag, and cannot trace $T_{i}$. Since each session of the protocol is randomized by the reader and the tag, it is not possible to trace the tag by analyzing the sent messages. Eventually, the improved scheme is forward untraceable and backward untraceable in this section.

\subsection{The Comparisons of Security Properties}

It can be seen from Table 2 that the improved protocol meets all security requirements compared with other schemes.

Table 2. The Comparisons of Security Properties

\begin{tabular}{llllll}
\hline Protocol & S1 & S2 & S3 & S4 & S5 \\
\hline Yoon et al.'s [10] & NO & NO & NO & NO & NO \\
Dhal et al.'s [11] & NO & NO & NO & NO & NO \\
Mohammadali et al.'s [12] & NO & NO & YES & NO & NO \\
Alakrut et al.'s [13] & NO & NO & NO & NO & NO \\
Karda s et al.'s [8] & YES & YES & NO & NO & NO \\
ours & YES & YES & YES & YES & YES \\
\hline
\end{tabular}

S1 : Tag impersonation resistance; S2 : DoS attack resistance; S3 : Secret disclosure and replay attack resistance; S4: forward untraceable;S5: backward untraceable.

\section{Conclusions}

Recently, the schemes $[8,14]$ have security issue. Although their protocol designers have claimed the optimal security for the protocol, our security analysis demonstrates that these protocols have serious disadvantage. Finally, we present a new ownership transfer protocol based on [8], which meets security requirements, such as tag impersonation resistance, DoS attack resistance, forward untraceable, backward untraceable, secret disclosure and replay attack resistance.

\section{Acknowledgments}

This work is supported in part by the 333 Project of Jiangsu Province (No. BRA2014047), and the Six Talent Peak Project of Jiangsu Province (2014-WLW-023).

\section{References}

[1] L. F. Shangguan, Z. J. Li, Z. Yang, M. Li, Y. H. Liu. OTrack: Order Tracking for Luggage in Mobile RFID Systems [M]. 2013 Proceedings Ieee Infocom. (2013), pp 3066-3074.

[2] E. W. T. Ngai, B. K. S. Cheung, S. S. Lam, and C. T. Ng. RFID value in aircraft parts supply chains: A case study [J]. International Journal of Production Economics, 147(2014), pp 330-339.

[3] S. F. Wamba. RFID-Enabled Healthcare Applications, Issues and Benefits: An Archival Analysis (1997-2011) [J]. Journal of medical systems, 36 (6) (2012) , pp 3393-3398.

[4] X. Chen, T. Cao, Guo Y. A new scalable RFID delegation protocol [J]. Appl Math, 8 (4) (2014), pp 1917-1924.

[5] H.-C. Lee, T. Eom, J. H. Yi. Secure and Lightweight Authentication Protocol for Mobile RFID Privacy [J]. Appl Math, 7 (1) (2013), pp 421-426.

[6] A. Fernàndez-Mir, R. Trujillo-Rasua, J. Castella-Roca, and J. Domingo-Ferrer. A scalable RFID authentication protocol supporting ownership transfer and controlled delegation [M]. RFID Security and Privacy. Springer. (2012),pp 147-162.

[7] M. R. Alagheband, M. R. Aref. Simulation-Based Traceability Analysis of RFID Authentication Protocols[J]. Wireless Personal Communications, (2013), pp 1-20. 
[8] S. Kardaş, S. Çelik, A. Arslan, and L. Albert. An efficient and private RFID authentication protocol supporting ownership transfer [M]. Lightweight Cryptography for Security and Privacy. Springer. (2013), pp 130-141.

[9] S. Vaudenay. On privacy models for RFID [M]. Advances in Cryptology-ASIACRYPT 2007. Springer. (2007),pp 68-87.

[10] E. J. Yoon. Improvement of the securing RFID systems conforming to EPC Class 1 Generation 2 standard [J]. Expert Systems with Applications, 39 (1) (2012) ,pp 1589-1594.

[11] S. Dhal, Sengupta I. Handling Authentication and Detection Probability in Multi-tag RFID Environment. Cryptology ePrint Archive, Report 2013/486, (2013), pp1-20.

[12] ] A. Mohammadali, Z. Ahmadian, Aref M. R. Analysis and Improvement of the securing RFID systems conforming to EPC Class 1 Generation 2 standard. IACR Cryptology ePrint Archive, (2013),pp 1-10.

[13] R. H. E. Alakrut, A. Samsudin, A. Syafalni. Provably Lightweight RFID Mutual Authentication Protocol. International Journal of Security \& Its Applications, 7 (4) (2013), pp 71-87.

[14] R. Doss, S. Sundaresan, W. Zhou, "Practical quadratic residues based scheme for authentication and privacy in mobile RFID systems. Ad Hoc Networks, 11 (1) (2013), pp 383-396.

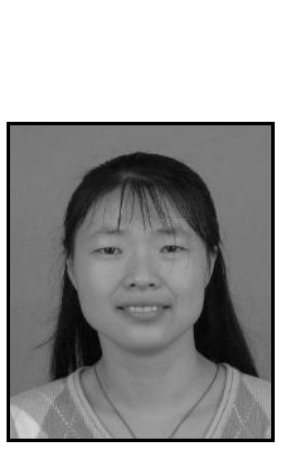

\begin{abstract}
Author
Xiuqing Chen, she received her bachelor's degree and master's degree from the China University of Mining and Technology. She had been a Ph.D. degree candidate in Computer Software and Theory from the China University of Mining and Technology. She has been a teacher in Xuzhou Medical College. Her research interests include security protocols and network security.
\end{abstract}


International Journal of Security and Its Applications

Vol.9, No.12 (2015) 